\title{
Gold nanoparticle-PPE constructs as biomolecular material mimics: Understanding the electrostatic and hydrophobic interactions
}

Ronnie L. Phillips, ${ }^{\dagger}$ Oscar R. Miranda, ${ }^{\ddagger}$ David E. Mortenson, ${ }^{\ddagger}$ Chandramouleeswaran Subramani, ${ }^{\ddagger}$ Vincent M. Rotello, ${ }^{*} \neq$ Uwe H.F. Bunz ${ }^{*, \dagger}$

${ }^{\dagger}$ School of Chemistry and Biochemistry, Georgia Institute of Technology, 901 Atlantic Drive, Atlanta, Georgia 30332

${ }^{\ddagger}$ Department of chemistry, University of Massachusetts, 710 North Pleasant Street, Amherst, Massachusetts 01003

E-mail: uwe.bunz@chemistry.gatech.edu; rotello@chem.umass.edu

Materials and Methods: Swallowtail-substituted carboxylate PPE (Sw-CO $\left.{ }_{2}\right)$ was synthesized according to published procedure. ${ }^{1}$ Fluorescence intensity changes at $465 \mathrm{~nm}$ were recorded in 96-well plates $\left(300 \mu \mathrm{L}\right.$ Whatman ${ }^{\circledR}$ Glass Bottom microplate) on a Molecular Devices SpectraMax M5 micro plate reader with an excitation wavelength of 405 nm. Phosphate buffer (PB), phosphate buffered saline (PBS), piperazine-1,4-bis(2ethanesulfonic acid) (PIPES), N-2-hydroxyethylpiperazine-N'-2-ethanesulfonic acid (HEPES), and tris(hydroxymethyl)aminomethane hydrochloride (Tris- $\mathrm{HCl}$ ) were purchased from Sigma-Aldrich. The buffers were diluted to a concentration of $5 \mathrm{mM}$ and a $\mathrm{pH}$ of 7.2 with DI $\mathrm{H}_{2} \mathrm{O}$. To calculate $\log$ P, computational program Maestro 8.0 was used. 
Scheme S1. Synthesis of Ligands ${ }^{2}$
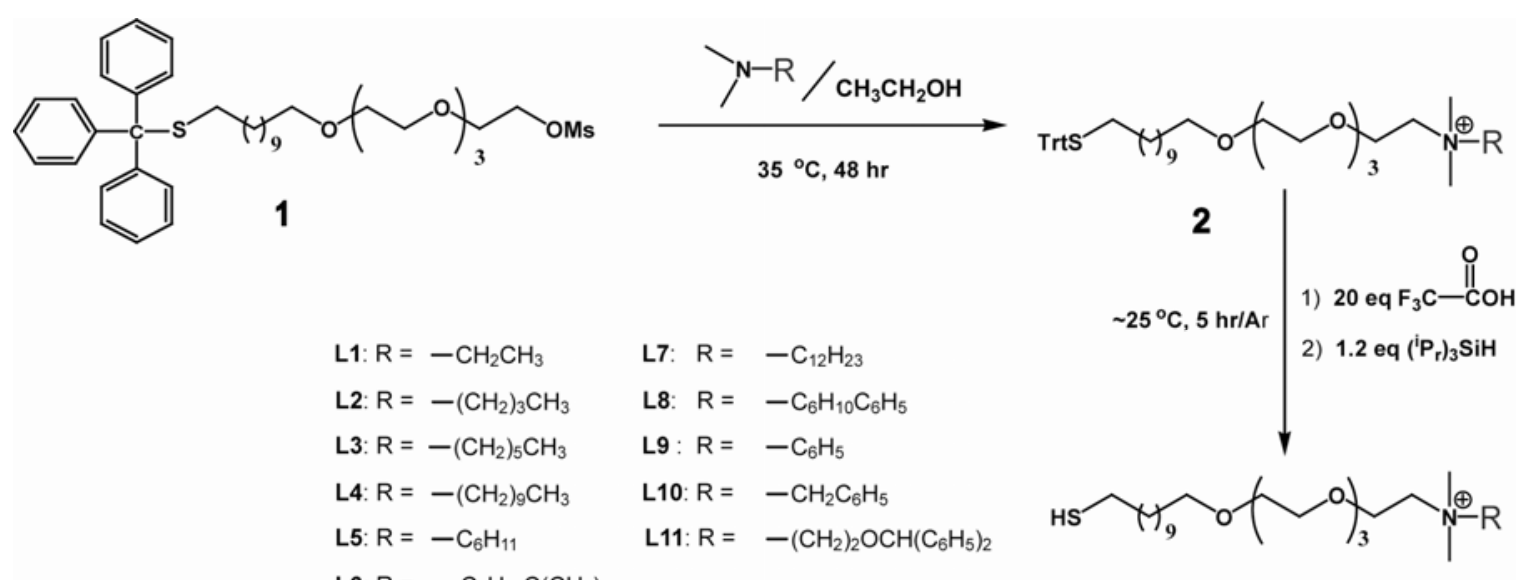

L1: $\mathrm{R}=-\mathrm{CH}_{2} \mathrm{CH}_{3}$
L2: $\mathrm{R}=-\left(\mathrm{CH}_{2}\right)_{3} \mathrm{CH}_{3}$
L3: $\mathrm{R}=-\left(\mathrm{CH}_{2}\right)_{5} \mathrm{CH}_{3}$
L4: $\mathrm{R}=-\left(\mathrm{CH}_{2}\right)_{9} \mathrm{CH}_{3}$
L5: $\mathrm{R}=-\mathrm{C}_{6} \mathrm{H}_{11}$
L6: $\mathrm{R}=-\mathrm{C}_{6} \mathrm{H}_{10} \mathrm{C}\left(\mathrm{CH}_{3}\right)_{3}$

$\mathbf{L}$

General procedure: Compound 2 bearing ammonium end groups were synthesized through the reaction of 1,1,1-triphenyl-14,17,20,23-tetraoxa-2- thiapentacosan-25-yl methanesulphonate (1) with corresponding substituted N,N-dimethylamines during $48 \mathrm{~h}$ at $\sim 35^{\circ} \mathrm{C}$. The trityl protected thiol ligand (2) was dissolved in dry dichloromethane (Methylene Chloride, DCM) and an excess of trifluoroacetic acid (TFA, $\sim 20$ equivalents) was added. The color of the solution was turned to yellow immediately. Subsequently, triisopropylsilane (TIPS, $\sim 1.2$ equivalents) was added to the reaction mixture. The reaction mixture was stirred for $\sim 5 \mathrm{~h}$ under Ar condition at room temperature. The solvent and most TFA and TIPS were distilled off under reduced pressure. The pale yellow residue was further dried in high vacuum. The product (L) formation was quantitative and their structure was confirmed by NMR. The yields were $>95 \%$.

Compound L1: ${ }^{1} \mathrm{H}$ NMR $\left(400 \mathrm{MHz}, \mathrm{CDCl}_{3}, \mathrm{TMS}\right): \delta 3.94\left(\mathrm{br}, 2 \mathrm{H},-\mathrm{OCH}_{2}-\left(\mathrm{CH}_{2} \mathrm{~N}\right)-\right), 3.69-3.56(\mathrm{~m}$, $\left.14 \mathrm{H},-\mathrm{CH}_{2} \mathrm{O}-+{ }_{-} \mathrm{CH}_{2} \mathrm{~N}-\right), 3.44\left(\mathrm{t}, 2 \mathrm{H},-\mathrm{CH}_{2} \mathrm{O}-\right), 3.40-3.32\left(\mathrm{~m}, 2 \mathrm{H},-\mathrm{NCH}_{2}-\right), 3.23\left(\mathrm{~s}, 6 \mathrm{H},-\left(\mathrm{CH}_{3}\right)_{2} \mathrm{~N}-\right)$, $2.78\left(\mathrm{~s}, 3 \mathrm{H},-\mathrm{CH}_{3} \mathrm{SO}_{3}^{-}\right), 2.51\left(\mathrm{q}, 2 \mathrm{H},-\mathrm{CH}_{2} \mathrm{~S}-\right), 1.69-1.149\left(\mathrm{~m}, 4 \mathrm{H},\left(\mathrm{SCH}_{2}\right) \underline{C}_{2}+-\mathrm{C}_{2}\left(\mathrm{CH}_{2} \mathrm{O}\right)-\right)$, 1.44-1.24 (m, $\left.18 \mathrm{H},-\mathrm{SH}+-\mathrm{CH}_{2^{-}}+-\left(\mathrm{NCH}_{2}\right) \mathrm{CH}_{3}\right)$.

Compound L2: ${ }^{1} \mathrm{H}$ NMR (400MHz, $\left.\mathrm{CDCl}_{3}, \mathrm{TMS}\right): \delta 3.96$ (br, $\left.2 \mathrm{H},-\mathrm{OCH}_{2}-\left(\mathrm{CH}_{2} \mathrm{~N}\right)-\right), 3.68-3.57$ (m, $\left.14 \mathrm{H},-\mathrm{CH}_{2} \mathrm{O}-+{ }_{-} \mathrm{CH}_{2} \mathrm{~N}-\right), 3.49$ (t, $\left.2 \mathrm{H},-\mathrm{CH}_{2} \mathrm{O}-\right), 3.39-3.33\left(\mathrm{~m}, 2 \mathrm{H},-\mathrm{NCH}_{2}-\right), 3.17\left(\mathrm{~s}, 6 \mathrm{H},-\left(\mathrm{CH}_{3}\right)_{2} \mathrm{~N}-\right)$, $2.91\left(\mathrm{~s}, 3 \mathrm{H},-\mathrm{CH}_{3} \mathrm{SO}_{3}^{-}\right), 2.52\left(\mathrm{q}, 2 \mathrm{H},-\mathrm{CH}_{2} \mathrm{~S}-\right), 1.78-1.52\left(\mathrm{~m}, 6 \mathrm{H},-\left(\mathrm{NCH}_{2}\right) \mathrm{C}_{2}{ }^{-}\right)+\left(\mathrm{SCH}_{2}\right) \mathrm{CH}_{2}+-$ $\left.\left.\mathrm{C}_{2}\left(\mathrm{CH}_{2} \mathrm{O}\right)-\right), 1.44-1.24\left(\mathrm{~m}, 17 \mathrm{H},-\mathrm{SH}+-\left(\mathrm{NCH}_{2} \mathrm{CH}_{2}-\right) \mathrm{CH}_{2}-\right)+-\mathrm{CH}_{2}-\right), 0.98\left(\mathrm{t}, 3 \mathrm{H},-\mathrm{CH}_{3}-\right)$.

Compound L3: ${ }^{1} \mathrm{H}$ NMR (400MHz, $\mathrm{CDCl}_{3}$, TMS): $\delta 3.95$ (br, $\left.2 \mathrm{H},-\mathrm{OC}_{2}-\left(\mathrm{CH}_{2} \mathrm{~N}\right)-\right)$, 3.68-3.56 (m, $\left.14 \mathrm{H},-\mathrm{CH}_{2} \mathrm{O}-+-\mathrm{CH}_{2} \mathrm{~N}-\right), 3.46$ (t, $\left.2 \mathrm{H},-\mathrm{CH}_{2} \mathrm{O}-\right)$, 3.40-3.33 (m, $\left.2 \mathrm{H},-\mathrm{NCH}_{2}-\right), 3.19\left(\mathrm{~s}, 6 \mathrm{H},-\left(\mathrm{CH}_{3}\right)_{2} \mathrm{~N}-\right)$, $\left.2.87\left(\mathrm{~s}, 3 \mathrm{H},-\mathrm{CH}_{3} \mathrm{SO}_{3}^{-}\right), 2.52\left(\mathrm{q}, 2 \mathrm{H},-\mathrm{CH}_{2} \mathrm{~S}-\right), 1.76-1.53\left(\mathrm{~m}, 6 \mathrm{H},-\left(\mathrm{NCH}_{2}\right) \mathrm{CH}_{2}\right)^{-}\right)+\left(\mathrm{SCH}_{2}\right) \mathrm{CH}_{2}+-$ $\left.\mathrm{C}_{2}\left(\mathrm{CH}_{2} \mathrm{O}\right)-\right)$, 1.41-1.22 (m, 21H, $\left.\left.-\mathrm{SH}+-\left(\mathrm{NCH}_{2} \mathrm{CH}_{2^{-}}\right) \mathrm{C}_{2^{-}}\right)+-\mathrm{CH}_{2^{-}}\right), 0.89$ (t, 3H, $\left.-\mathrm{CH}_{3^{-}}\right)$.

Compound L4: ${ }^{1} \mathrm{H}$ NMR (400MHz, $\left.\mathrm{CDCl}_{3}, \mathrm{TMS}\right): \delta 3.94$ (br, 2H, - $\left.\mathrm{OCH}_{2}-\left(\mathrm{CH}_{2} \mathrm{~N}\right)-\right), 3.67-3.54(\mathrm{~m}$, $\left.14 \mathrm{H},-\mathrm{CH}_{2} \mathrm{O}-+{ }_{-} \mathrm{CH}_{2} \mathrm{~N}-\right), 3.48\left(\mathrm{t}, 2 \mathrm{H},-\mathrm{CH}_{2} \mathrm{O}-\right), 3.41-3.32\left(\mathrm{~m}, 2 \mathrm{H},-\mathrm{NCH}_{2}-\right), 3.17\left(\mathrm{~s}, 6 \mathrm{H},-\left(\mathrm{CH}_{3}\right)_{2} \mathrm{~N}-\right)$, $2.90\left(\mathrm{~s}, 3 \mathrm{H},-\mathrm{CH}_{3} \mathrm{SO}_{3}^{-}\right), 2.51\left(\mathrm{q}, 2 \mathrm{H},-\mathrm{CH}_{2} \mathrm{~S}-\right), 1.78-1.52\left(\mathrm{~m}, 6 \mathrm{H},-\left(\mathrm{NCH}_{2}\right) \mathrm{CH}_{2}-\right)+\left(\mathrm{SCH}_{2}\right) \mathrm{C}_{2}+-$ $\left.\left.\mathrm{C}_{2}\left(\mathrm{CH}_{2} \mathrm{O}\right)-\right), 1.45-1.15\left(\mathrm{~m}, 29 \mathrm{H},-\mathrm{SH}+-\left(\mathrm{NCH}_{2} \mathrm{CH}_{2^{-}}\right) \mathrm{C}_{2}{ }^{-}\right)+-\mathrm{CH}_{2}-\right), 0.87$ (t, 3H, - $\left.\mathrm{CH}_{3}-\right)$.

Compound L5: ${ }^{1} \mathrm{H}$ NMR (400MHz, $\left.\mathrm{CDCl}_{3}, \mathrm{TMS}\right)$ : $\delta 3.95$ (br, $\left.2 \mathrm{H},-\mathrm{OCH}_{2}-\left(\mathrm{CH}_{2} \mathrm{~N}\right)-\right), 3.81-3.72(\mathrm{~m}$, $\left.1 \mathrm{H}, \mathrm{H}_{\text {Cyclo }}\right), 3.69-3.53\left(\mathrm{~m}, 14 \mathrm{H},-\mathrm{CH}_{2} \mathrm{O}-+-\mathrm{CH}_{2} \mathrm{~N}-\right), 3.49\left(\mathrm{t}, 2 \mathrm{H},-\mathrm{CH}_{2} \mathrm{O}-\right), 3.11\left(\mathrm{~s}, 6 \mathrm{H},-\left(\mathrm{CH}_{3}\right)_{2} \mathrm{~N}-\right)$, 
$2.91\left(\mathrm{~s}, 3 \mathrm{H},-\mathrm{CH}_{3} \mathrm{SO}_{3}^{-}\right.$), 2.52 (q, 2H, - $\left.\mathrm{CH}_{2} \mathrm{~S}-\right), 2.23$ (d, 2H, $\mathrm{H}_{\text {Cyclo }}$ ), 1.99 (d, 2H, $\mathrm{H}_{\text {Cyclo }}$ ), 1.78-1.52 (m, $\left.4 \mathrm{H},-\left(\mathrm{SCH}_{2}\right) \underline{\mathrm{C}}_{2}+-\mathrm{C}_{2}\left(\mathrm{CH}_{2} \mathrm{O}\right)-\right), 1.51-1.12\left(\mathrm{~m}, 21 \mathrm{H}, \mathrm{SH}+-\mathrm{CH} 2-+\mathrm{H}_{\mathrm{Cyclo}}\right)$.

Compound L6: ${ }^{1} \mathrm{H}$ NMR (400MHz, $\left.\mathrm{CDCl}_{3}, \mathrm{TMS}\right)$ : $\delta 3.96$ (br, $\left.2 \mathrm{H},-\mathrm{OC}_{2}-\left(\mathrm{CH}_{2} \mathrm{~N}\right)-\right)$, 3.79-3.75 (m, $\left.1 \mathrm{H}, \mathrm{H}_{\text {Cyclo }}\right), 3.66-3.57\left(\mathrm{~m}, 14 \mathrm{H},-\mathrm{CH}_{2} \mathrm{O}-+-\mathrm{CH}_{2} \mathrm{~N}-\right), 3.46\left(\mathrm{t}, 2 \mathrm{H},-\mathrm{CH}_{2} \mathrm{O}-\right), 3.12\left(\mathrm{~s}, 6 \mathrm{H},-\left(\mathrm{CH}_{3}\right)_{2} \mathrm{~N}-\right)$, 2.89 (s, 3H, - $\mathrm{CH}_{3} \mathrm{SO}_{3}^{-}$), 2.52 (q, 2H, $\left.-\mathrm{CH}_{2} \mathrm{~S}-\right), 2.28$ (d, 2H, $\mathrm{H}_{\text {Cyclo }}$ ), 2.01 (d, 2H, $\mathrm{H}_{\text {Cyclo }}$ ), 1.64-1.54 (m, $\left.4 \mathrm{H},-\left(\mathrm{SCH}_{2}\right) \underline{\mathrm{C}}_{2}+-\mathrm{C}_{2}\left(\mathrm{CH}_{2} \mathrm{O}\right)-\right), 1.47\left(\mathrm{q}, 2 \mathrm{H}, \mathrm{H}_{\text {Cyclo }}\right), 1.33\left(\mathrm{t},{ }^{3} \mathrm{~J}=8.0 \mathrm{~Hz}, 1 \mathrm{H},-\mathrm{SH}\right), 1.30-1.22(\mathrm{~m}$, $14 \mathrm{H},-\mathrm{CH} 2-), 1.16$ (q, 2H, $\left.\mathrm{H}_{\mathrm{Cyclo}}\right) 1.04$ (td, $\left.\left.1 \mathrm{H}-\mathrm{CHC}-\right), 0.86\left(\mathrm{~s}, 9 \mathrm{H},-\mathrm{C}\left(\mathrm{CH}_{3}\right)_{3}\right)^{-}\right)$.

Compound L7: ${ }^{1} \mathrm{H}$ NMR $\left(400 \mathrm{MHz}, \mathrm{CDCl}_{3}, \mathrm{TMS}\right): \delta 3.98$ (br, $\left.2 \mathrm{H},-\mathrm{OCH}_{2}-\left(\mathrm{CH}_{2} \mathrm{~N}\right)-\right), 3.78-3.75(\mathrm{~m}$, $\left.1 \mathrm{H}, \mathrm{H}_{\text {Cyclo }}\right), 3.64-3.55\left(\mathrm{~m}, 14 \mathrm{H},-\mathrm{CH}_{2} \mathrm{O}-+\mathrm{CH}_{2} \mathrm{~N}-\right), 3.46-3.42\left(\mathrm{dt}, 2 \mathrm{H},-\mathrm{CH}_{2} \mathrm{O}-\right), 3.16(\mathrm{~s}, 6 \mathrm{H}$, $\left.\left(\mathrm{CH}_{3}\right)_{2} \mathrm{~N}-\right), 2.86\left(\mathrm{~s}, 3 \mathrm{H},-\mathrm{CH}_{3} \mathrm{SO}_{3}^{-}\right), 2.52$ (q, 2H, $\left.-\mathrm{CH}_{2} \mathrm{~S}-\right), 1.93-1.40\left(\mathrm{~m}, 26 \mathrm{H}, \mathrm{SCH}_{2}\right) \mathrm{CH}_{2}+-$ $\left.\mathrm{C}_{2}\left(\mathrm{CH}_{2} \mathrm{O}\right)-+\mathrm{H}_{\text {Cyclo }}\right), 1.33\left(\mathrm{t},{ }^{3} J=7.82 \mathrm{~Hz}, 1 \mathrm{H},-\mathrm{SH}\right), 1.29-1.24$ (m, 14H, -CH2-).

Compound L8: ${ }^{1} \mathrm{H}$ NMR (400MHz, $\mathrm{CDCl}_{3}$, TMS): $\delta$ 7.4-7.2 (m, 4H, $\left.\mathrm{H}_{\mathrm{Ar}}\right), 7.17\left(\mathrm{~d}, 1 \mathrm{H}, \mathrm{H}_{\mathrm{Ar}}\right), 3.95(\mathrm{~d}$ and br, $\left.2 \mathrm{H},-\mathrm{OCH}_{2}-\left(\mathrm{CH}_{2} \mathrm{~N}\right)-\right), 3.79-3.52\left(\mathrm{~m}, 14 \mathrm{H},-\mathrm{CH}_{2} \mathrm{O}-+-\mathrm{CH}_{2} \mathrm{~N}-\right), 3.45\left(\mathrm{q}, 2 \mathrm{H},-\mathrm{CH}_{2} \mathrm{O}-\right), 3.29-3.22$ ( $\mathrm{m}$ and br, $1 \mathrm{H}, \mathrm{H}_{\text {Cyclo }}$ ), 3.01-2.92 ( $\mathrm{m}$ and br, $\left.1 \mathrm{H}, \mathrm{H}_{\text {Cyclo }}\right) 2.87$ (s, 3H, $-\mathrm{CH}_{3} \mathrm{SO}_{3}^{-}$), 2.81 (d and br, 6H, $\left.\left(\mathrm{CH}_{3}\right)_{2} \mathrm{~N}-\right), 2.52$ (q, $\left.2 \mathrm{H},-\mathrm{CH}_{2} \mathrm{~S}-\right), 2.39-2.26\left(\mathrm{~m}, 2 \mathrm{H}, \mathrm{H}_{\text {Cyclo }}\right), 2.19-2.06\left(\mathrm{~m}, 2 \mathrm{H}, \mathrm{H}_{\text {Cyclo }}\right), 1.96-1.84(\mathrm{~m}$, $\left.4 \mathrm{H}, \mathrm{H}_{\text {Cyclo }}\right), 1.72-1.53\left(\mathrm{~m}, 4 \mathrm{H},-\left(\mathrm{SCH}_{2}\right) \mathrm{CH}_{2}+-\mathrm{CH}_{2}\left(\mathrm{CH}_{2} \mathrm{O}\right)-\right), 1.42-1.1 .19\left(\mathrm{~m}, 15 \mathrm{H},-\mathrm{SH}+-\mathrm{CH}_{2}-\right)$.

Compound L9: ${ }^{1} \mathrm{H}$ NMR (400MHz, $\mathrm{CDCl}_{3}$, TMS): $\delta 7.82\left(\mathrm{~d}, 2 \mathrm{H}, \mathrm{H}_{\mathrm{Ar}}\right), 7.66-7.51\left(\mathrm{~m}, 3 \mathrm{H}, \mathrm{H}_{\mathrm{Ar}}\right), 4.24$ (br, $\left.2 \mathrm{H},-\mathrm{OCH}_{2}-\left(\mathrm{CH}_{2} \mathrm{~N}\right)-\right), 3.78\left(\mathrm{~s}, 6 \mathrm{H},-\left(\mathrm{CH}_{3}\right)_{2} \mathrm{~N}-\right), 3.68-3.52\left(\mathrm{~m}, 14 \mathrm{H},-\mathrm{CH}_{2} \mathrm{O}-+-\mathrm{CH}_{2} \mathrm{~N}-\right)$, 3.47-3.36 $\left(\mathrm{m}, 2 \mathrm{H},-\mathrm{CH}_{2} \mathrm{O}-\right), 2.87\left(\mathrm{~s}, 3 \mathrm{H},-\mathrm{CH}_{3} \mathrm{SO}_{3}^{-}\right), 2.52$ (q, $\left.2 \mathrm{H},-\mathrm{CH}_{2} \mathrm{~S}-\right), 1.70-1.46\left(\mathrm{~m}, 4 \mathrm{H},-\left(\mathrm{SCH}_{2}\right) \mathrm{C}_{2}+-\right.$ $\left.\mathrm{C}_{2}\left(\mathrm{CH}_{2} \mathrm{O}\right)-\right), 1.42-1.1 .16\left(\mathrm{~m}, 15 \mathrm{H},-\mathrm{SH}+-\mathrm{CH}_{2}-\right)$.

Compound L10: ${ }^{1} \mathrm{H}$ NMR (400MHz, $\mathrm{CDCl}_{3}$, TMS): $\delta 8.37$ (d, $\left.1 \mathrm{H}, \mathrm{H}_{\mathrm{Ar}}\right), 7.98\left(\mathrm{~d}, 1 \mathrm{H}, \mathrm{H}_{\mathrm{Ar}}\right), 7.69-7.61$ $\left(\mathrm{m}, 3 \mathrm{H}, \mathrm{H}_{\mathrm{Ar}}\right), 7.59-7.48\left(\mathrm{~m}, 1 \mathrm{H}, \mathrm{H}_{\mathrm{Ar}}\right), 4.38$ (br, $\left.\left.2 \mathrm{H},-\mathrm{NCH}_{2}-\mathrm{Ar}\right)\right), 3.76\left(\mathrm{br}, 2 \mathrm{H},-\mathrm{OC}_{2}-\left(\mathrm{CH}_{2} \mathrm{~N}\right)-\right)$ 3.72$3.62\left(\mathrm{~m}, 14 \mathrm{H},-\mathrm{CH}_{2} \mathrm{O}-+-\mathrm{CH}_{2} \mathrm{~N}-\right), 3.61-3.55\left(\mathrm{~m}, 2 \mathrm{H},-\mathrm{CH}_{2} \mathrm{O}-\right), 3.23\left(\mathrm{~s}, 6 \mathrm{H},-\left(\mathrm{CH}_{3}\right)_{2} \mathrm{~N}-\right), 3.07$ (s, $3 \mathrm{H},-$ $\left.\mathrm{CH}_{3} \mathrm{SO}_{3}^{-}\right), 2.52$ (q, 2H, $\left.-\mathrm{CH}_{2} \mathrm{~S}-\right)$, 1.67-1.51 (m, 4H, - $\left.\left(\mathrm{SCH}_{2}\right) \mathrm{CH}_{2}+-\mathrm{CH}_{2}\left(\mathrm{CH}_{2} \mathrm{O}\right)-\right)$, 1.35-1.21 (m, $\left.15 \mathrm{H},-\mathrm{SH}+-\mathrm{CH}_{2}-\right)$.

Compound L11: ${ }^{1} \mathrm{H}$ NMR (400MHz, $\mathrm{CDCl}_{3}$, TMS): $\delta 7.42\left(\mathrm{~d}, 2 \mathrm{H}, \mathrm{H}_{\mathrm{Ar}}\right), 7.37-2.27\left(\mathrm{~m}, 8 \mathrm{H}, \mathrm{H}_{\mathrm{Ar}}\right), 7.25-$ $7.18\left(\mathrm{t}, 2 \mathrm{H}, \mathrm{H}_{\mathrm{Ar}}\right), 5.13\left(\mathrm{~s}, 1 \mathrm{H}, \mathrm{H}_{\mathrm{Ar}}\right), 4.12\left(\mathrm{br}, 2 \mathrm{H},-\mathrm{CH}_{2} \mathrm{OCAr}-\right), 3.96\left(\mathrm{br}, 2 \mathrm{H},-\mathrm{OCH}_{2}-\left(\mathrm{CH}_{2} \mathrm{~N}\right)-\right)$, 3.64$3.51\left(\mathrm{~m}, 14 \mathrm{H},-\mathrm{CH}_{2} \mathrm{O}-+-\mathrm{CH}_{2} \mathrm{~N}-\right), 3.45\left(\mathrm{t}, 2 \mathrm{H},-\mathrm{CH}_{2} \mathrm{O}-\right), 3.29-3.34\left(\mathrm{~m}, 2 \mathrm{H},-\mathrm{NCH}_{2}\left(\mathrm{CH}_{2} \mathrm{OCAr}\right), 3.28\right.$ $\left(\mathrm{s}, 6 \mathrm{H},-\left(\mathrm{CH}_{3}\right)_{2} \mathrm{~N}-\right), 2.86\left(\mathrm{~s}, 3 \mathrm{H},-\mathrm{CH}_{3} \mathrm{SO}_{3}^{-}\right), 2.52\left(\mathrm{q}, 2 \mathrm{H},-\mathrm{CH}_{2} \mathrm{~S}-\right), 1.60-1.48\left(\mathrm{~m}, 4 \mathrm{H},-\left(\mathrm{SCH}_{2}\right) \mathrm{CH}_{2}+-\right.$ $\left.\mathrm{C}_{2}\left(\mathrm{CH}_{2} \mathrm{O}\right)-\right), 1.34-1.16\left(\mathrm{~m}, 15 \mathrm{H},-\mathrm{SH}+-\mathrm{CH}_{2}-\right)$. 
Scheme S2. Synthesis of cationic gold nanoparticles $\left(\mathbf{N P}_{\mathbf{1}}-\mathbf{N P}_{11}\right)$

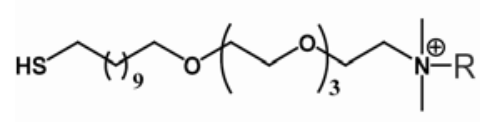

Ls

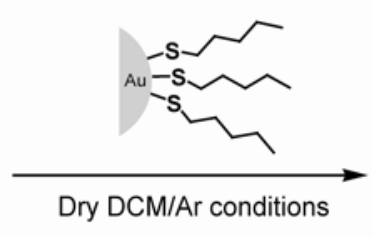

Dry DCM/Ar conditions

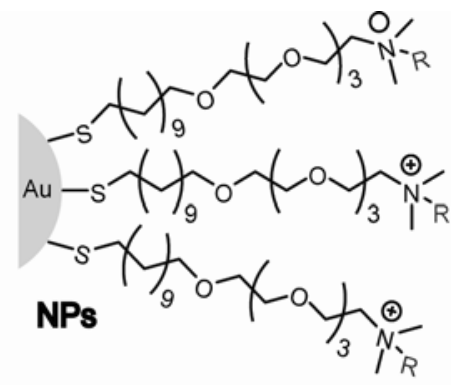

1-Pentanethiol coated gold nanoparticles $(d=\sim 2 \mathrm{~nm})$ were prepared according to the previously reported protocol. ${ }^{3}$ Place-exchange reaction ${ }^{4}$ of compound Ls dissolved in DCM with pentanethiol-coated gold nanoparticles $(\mathrm{d} \sim 2 \mathrm{~nm})$ was carried out for 3 days at environmental temperature. Then, DCM was evaporated under reduced pressure. The residue was dissolved in a small amount of distilled water and dialyzed (membrane $\mathrm{MWCO}=1,000)$ to remove excess ligands, acetic acid and the other salts present with the nanoparticles. After dialysis, the particles were lyophilized to afford a brownish solid. The particles (NPs) are redispersed in water and/or ionized water $(18 \mathrm{M} \Omega-\mathrm{cm}) .{ }^{1} \mathrm{H}$ NMR spectra in $\mathrm{D}_{2} \mathrm{O}$ showed substantial broadening of the proton signals and no free ligands were observed. 

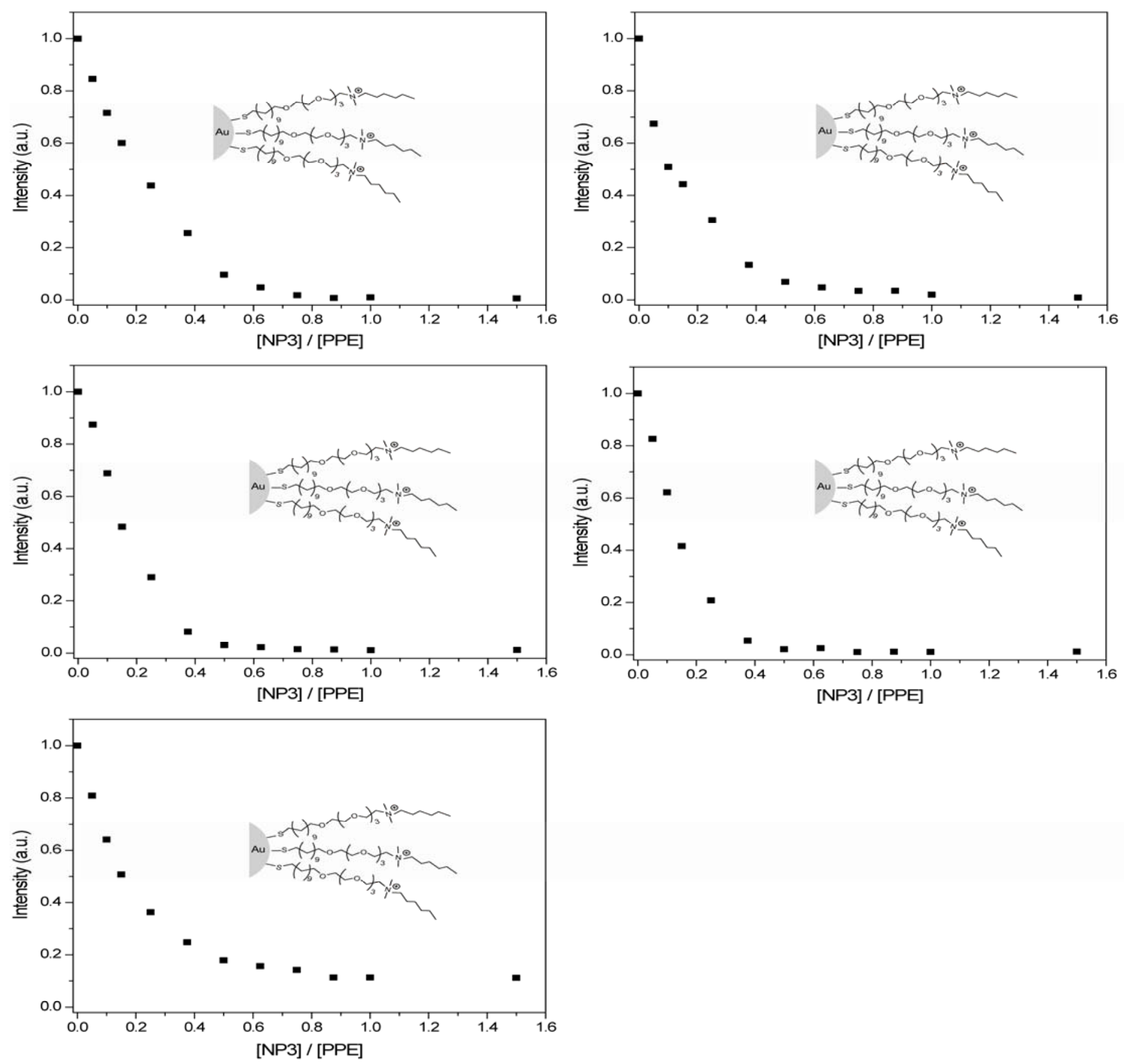

Figure S1. Fluorescence titration curves for the complexation of $\mathbf{N P}_{3}$ with $\mathbf{S w}-\mathbf{C O}_{2}$ in $\mathrm{PB}$ (top left), PIPES (top right), HEPES (middle left), Tris- $\mathrm{HCl}$ (middle right), and PBS (bottom left). The changes in fluorescence intensity at $465 \mathrm{~nm}$ were measured following the addition of cationic nanoparticles with an excitation wavelength of $405 \mathrm{~nm}$. The complexation of $\mathbf{N P}_{3}$ with $\mathbf{S w}-\mathbf{C O}_{2}$ in all buffers was similar except in PBS, which disrupted the complexation due to the salt concentration. 

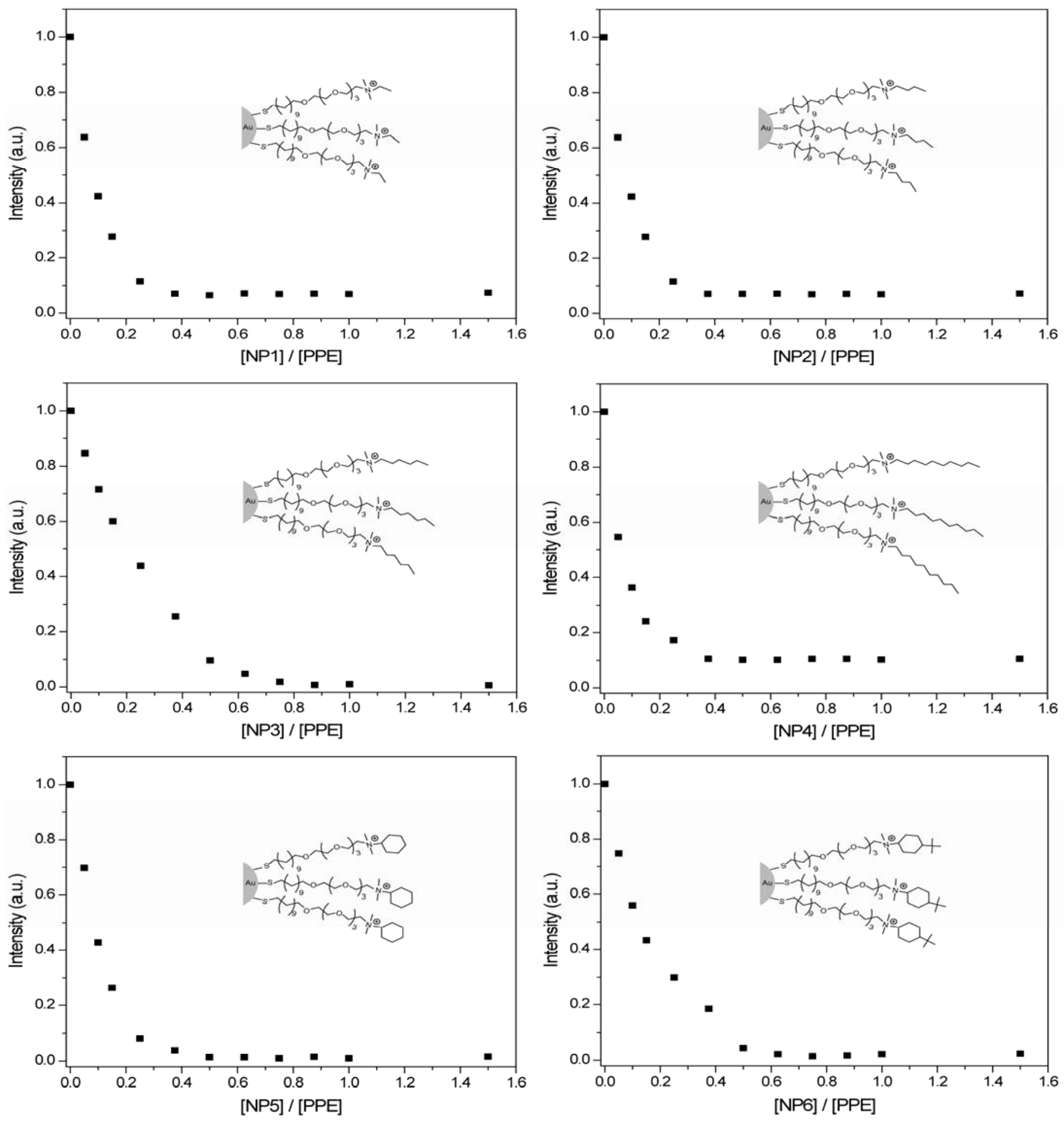

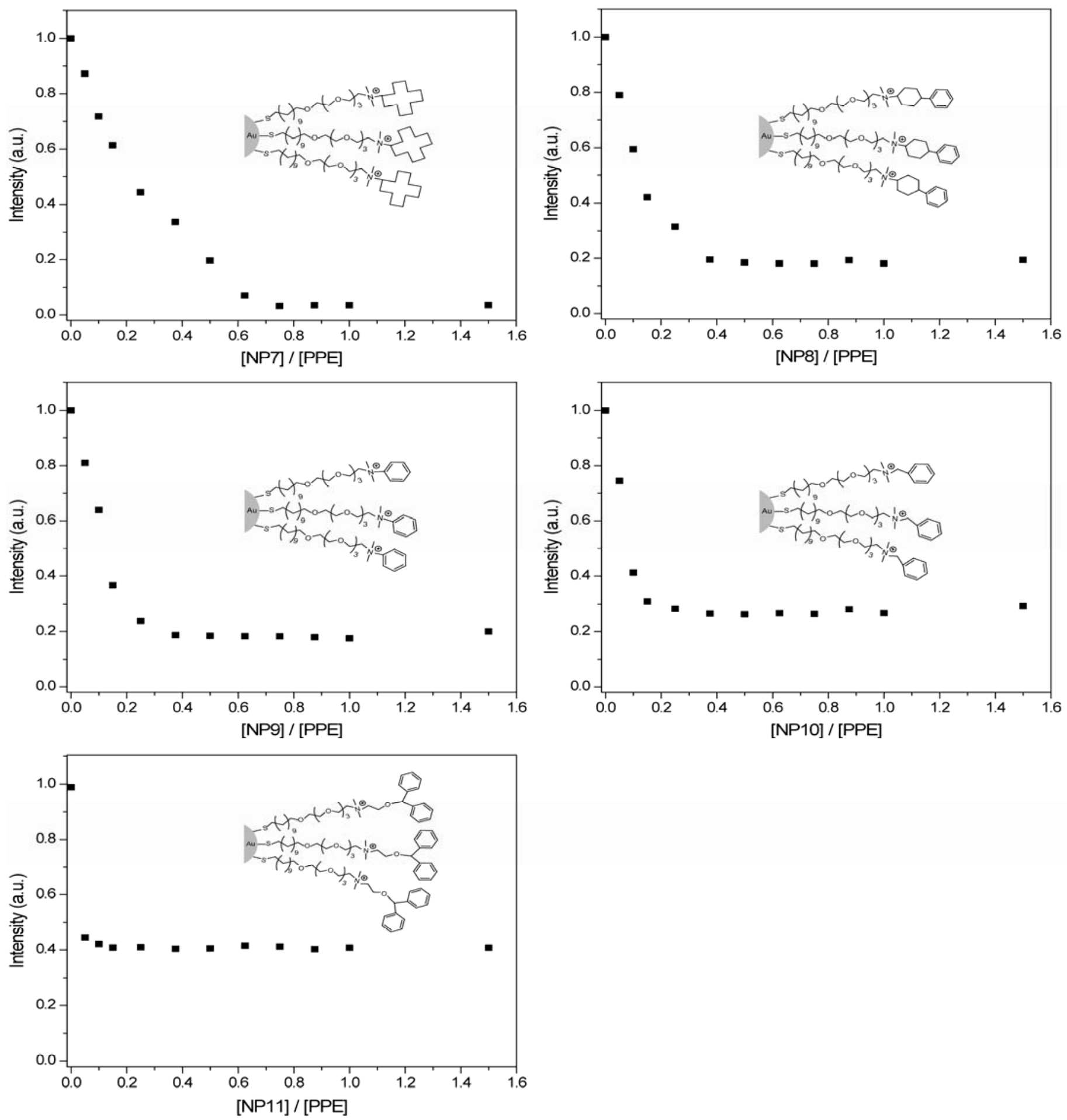

Figure S2. Fluorescence titration curves for the complexation of $\mathbf{N P}_{\mathbf{1}}-\mathbf{N P}_{\mathbf{1 1}}$ with $\mathbf{S w}-\mathbf{C O}_{\mathbf{2}}$ in $\mathrm{PB}$ with no $\mathrm{NaCl}$. The changes in fluorescence intensity at $465 \mathrm{~nm}$ were measured following the addition of cationic nanoparticles with an excitation wavelength of $405 \mathrm{~nm}$. 

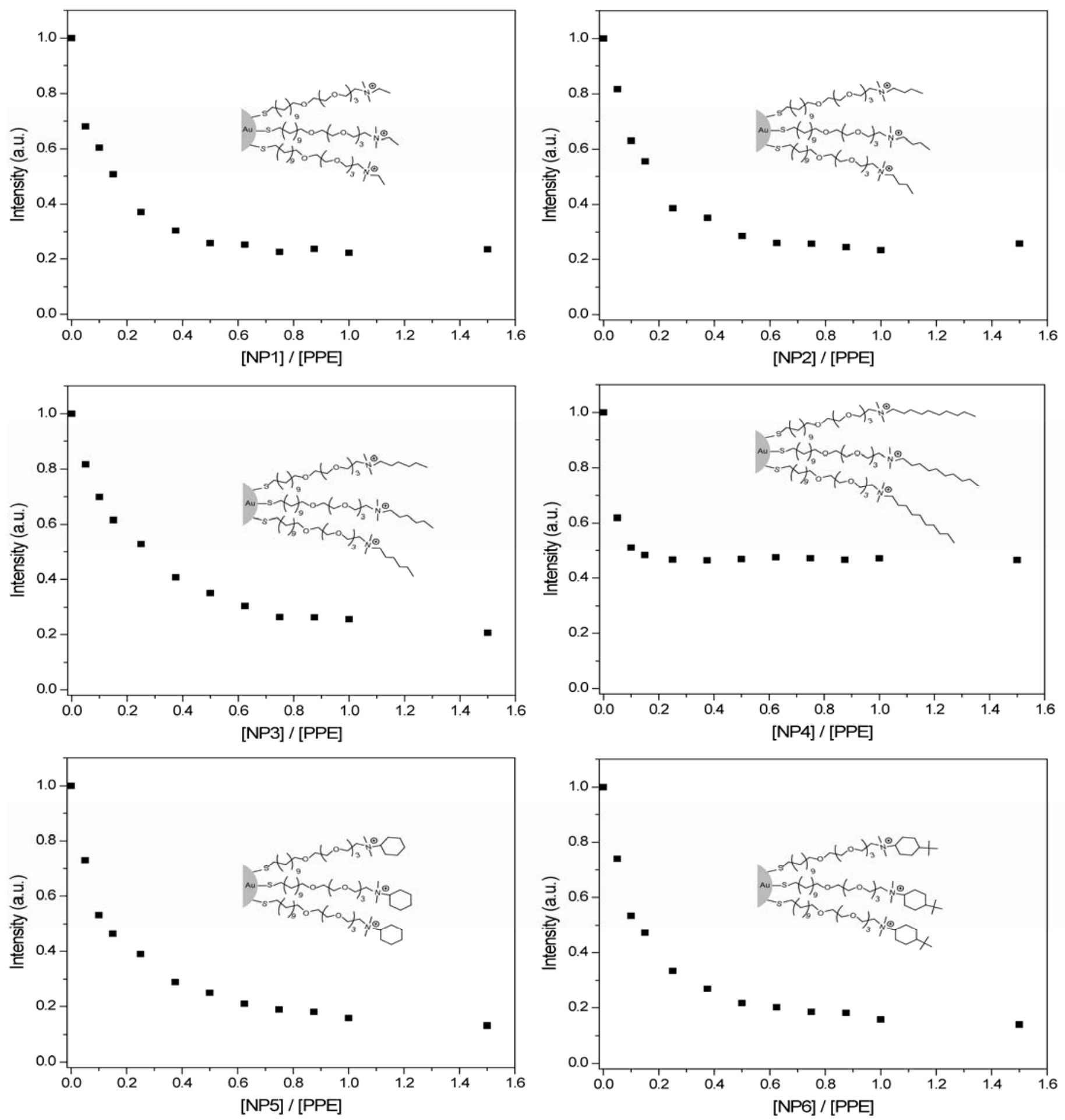

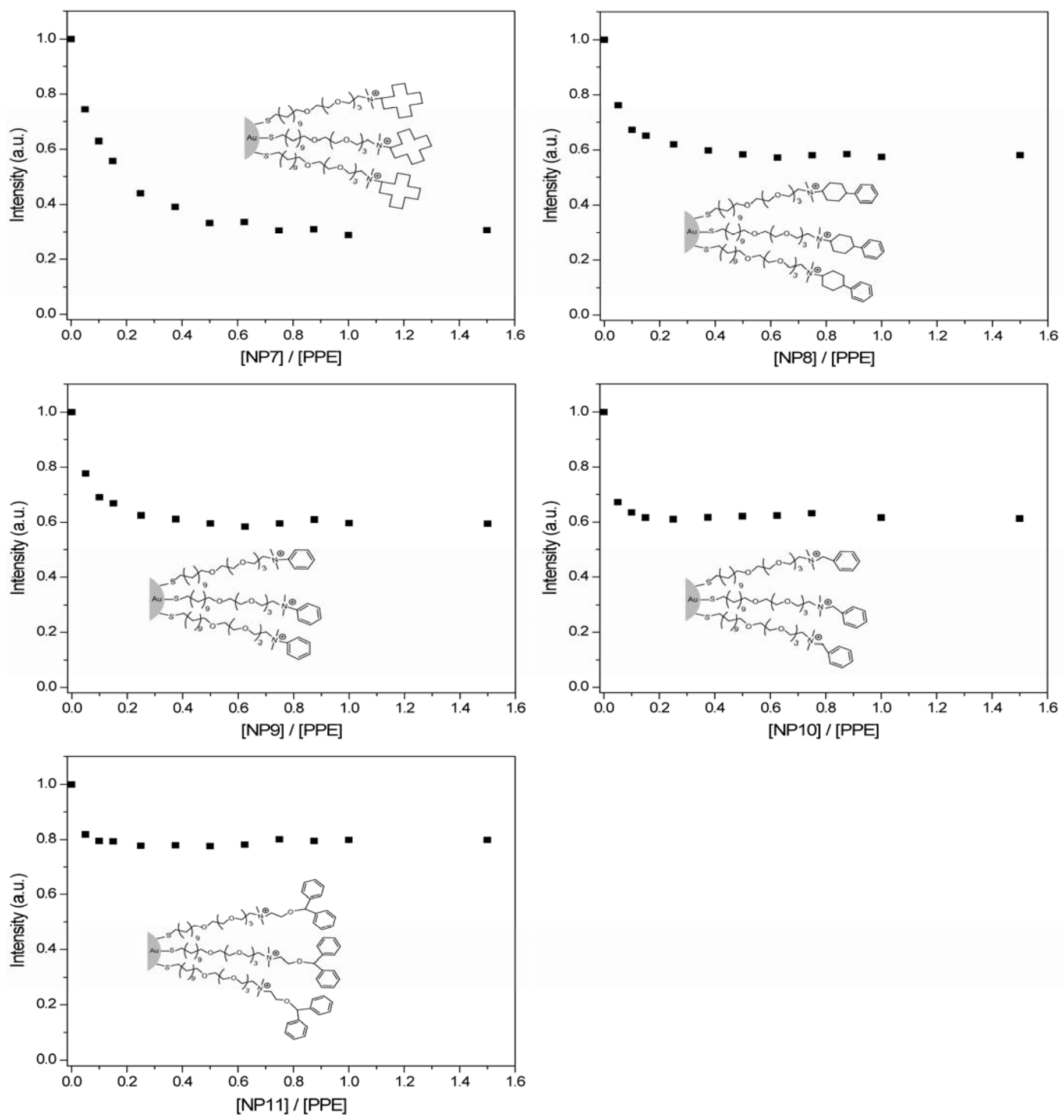

Figure S3. Fluorescence titration curves for the complexation of $\mathbf{N P}_{\mathbf{1}}-\mathbf{N P}_{\mathbf{1 1}}$ with $\mathbf{S w}-\mathbf{C O}_{\mathbf{2}}$ in PB with $100 \mathrm{mM} \mathrm{NaCl}$. The changes in fluorescence intensity at $465 \mathrm{~nm}$ were measured following the addition of cationic nanoparticles with an excitation wavelength of $405 \mathrm{~nm}$. 

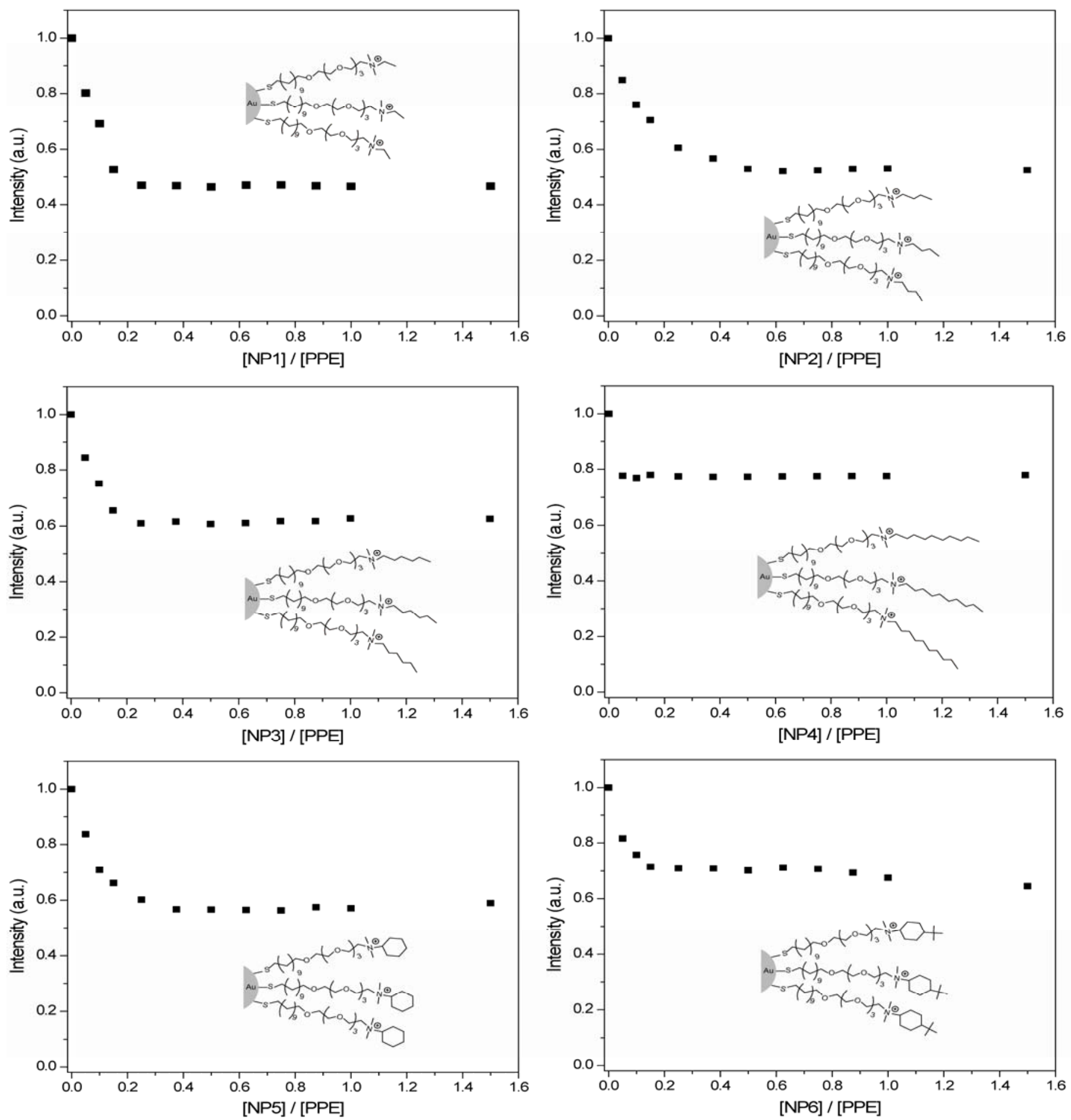

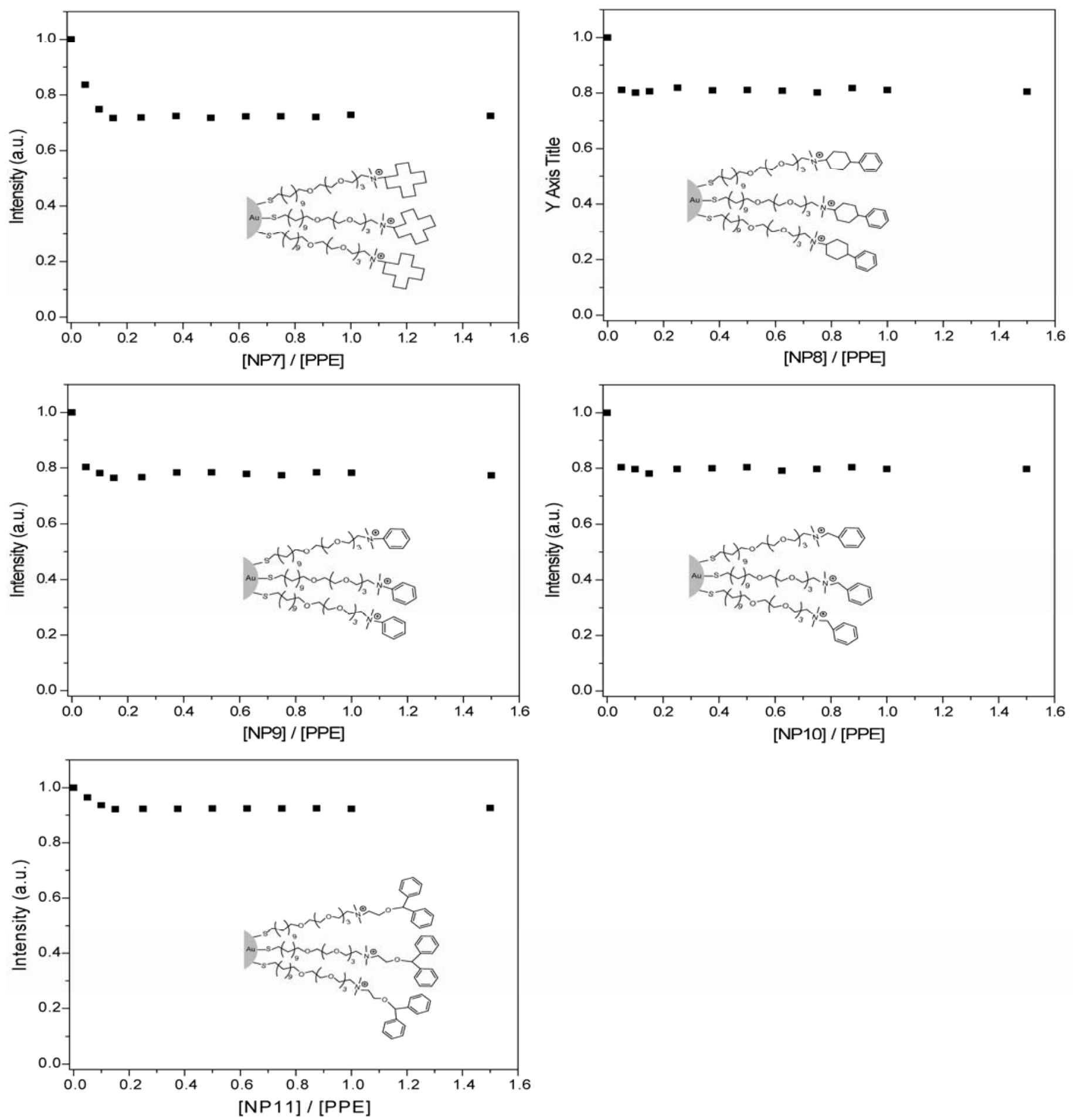

Figure S4. Fluorescence titration curves for the complexation of $\mathbf{N P}_{\mathbf{1}}-\mathbf{N P}_{\mathbf{1 1}}$ with $\mathbf{S w}-\mathbf{C O}_{\mathbf{2}}$ in $\mathrm{PB}$ with $250 \mathrm{mM} \mathrm{NaCl}$. The changes in fluorescence intensity at $465 \mathrm{~nm}$ were measured following the addition of cationic nanoparticles with an excitation wavelength of $405 \mathrm{~nm}$. 

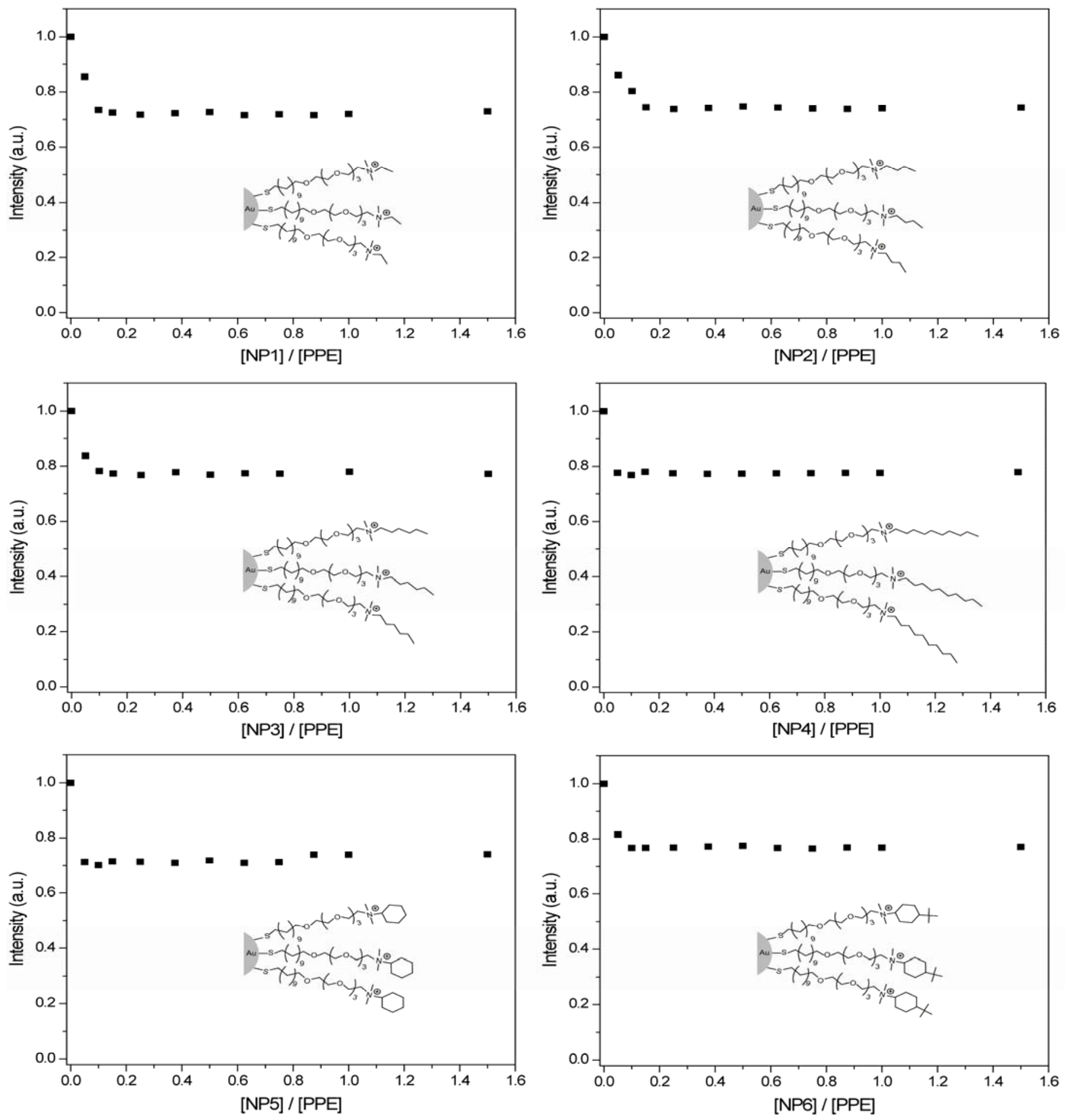

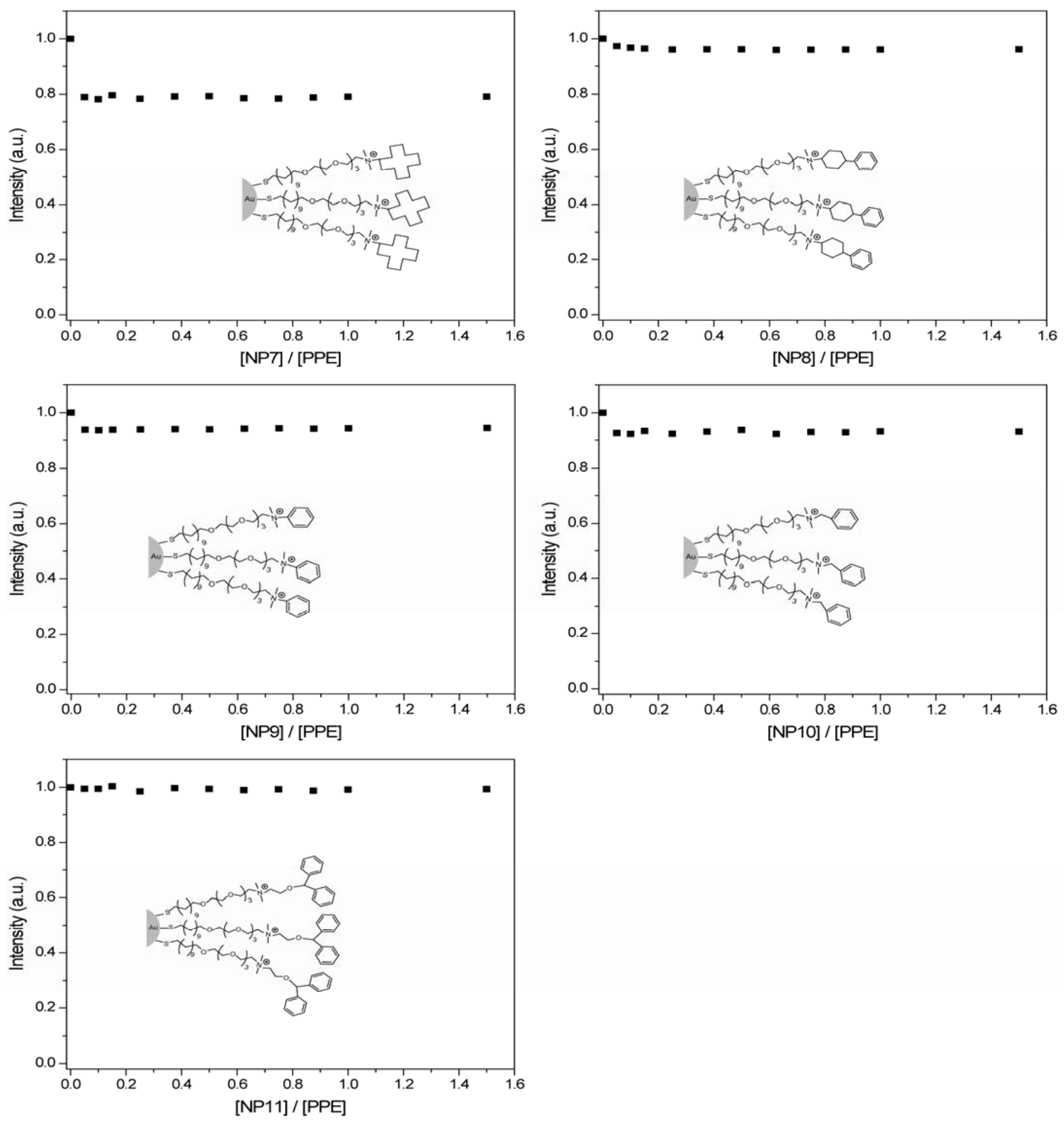

Figure S5. Fluorescence titration curves for the complexation of $\mathbf{N P}_{\mathbf{1}}-\mathbf{N P}_{\mathbf{1 1}}$ with $\mathbf{S} \mathbf{w}-\mathbf{C O}_{2}$ in PB with $500 \mathrm{mM} \mathrm{NaCl}$. The changes in fluorescence intensity at $465 \mathrm{~nm}$ were measured following the addition of cationic nanoparticles with an excitation wavelength of $405 \mathrm{~nm}$. 

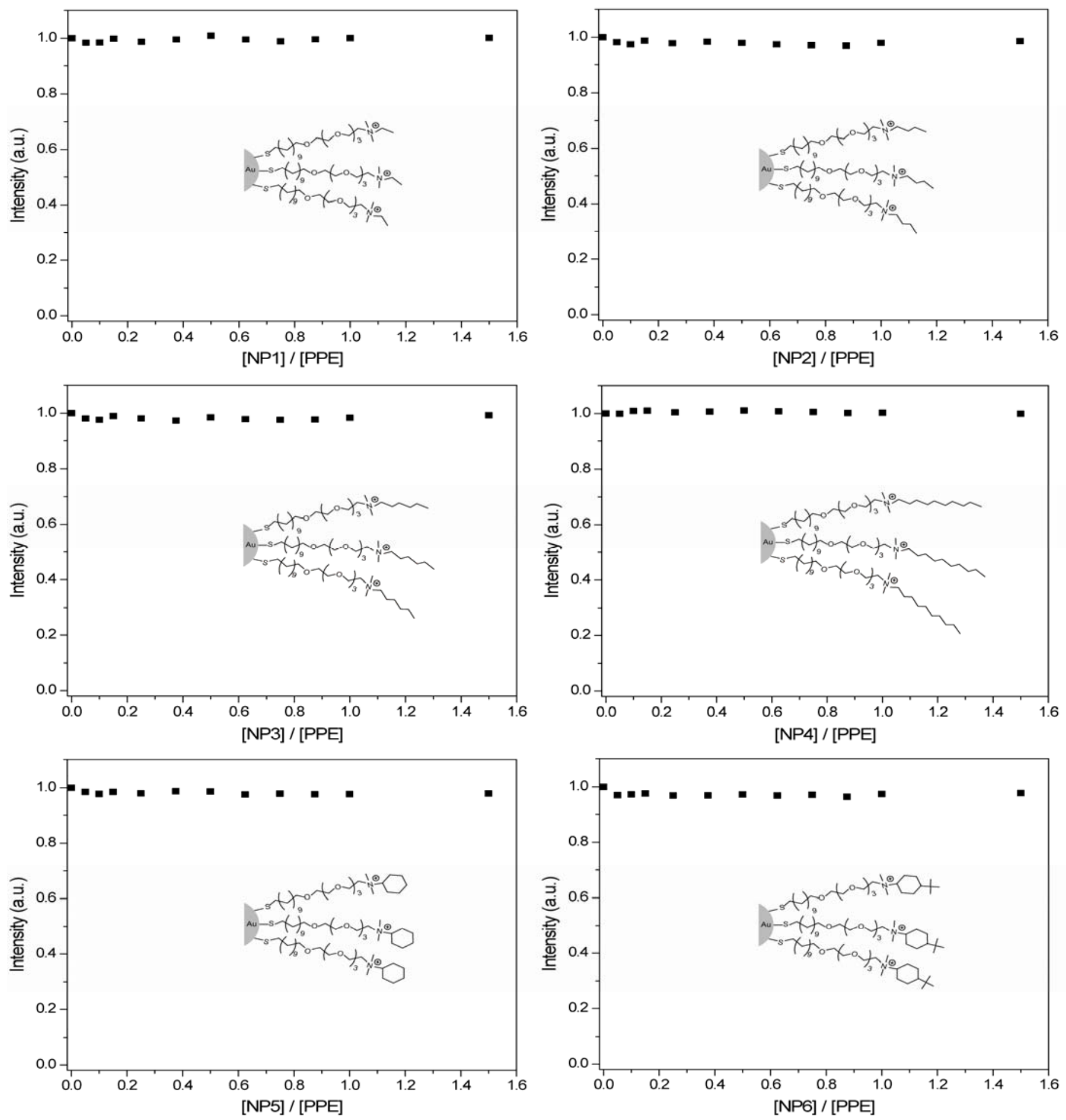

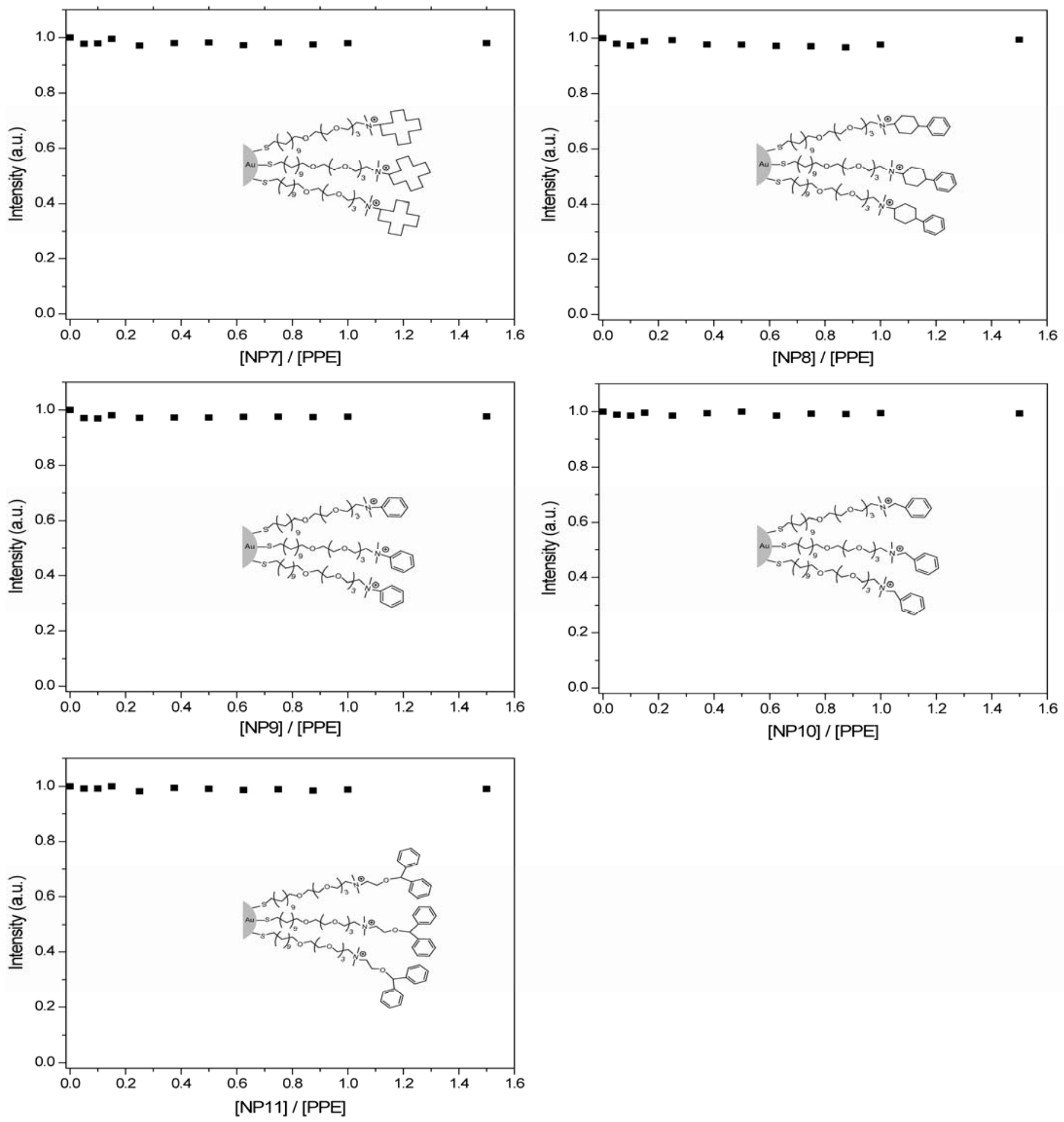

Figure S6. Fluorescence titration curves for the complexation of $\mathbf{N P}_{\mathbf{1}}-\mathbf{N P}_{\mathbf{1 1}}$ with $\mathbf{S w}-\mathbf{C O}_{\mathbf{2}}$ in PB with $1000 \mathrm{mM} \mathrm{NaCl}$. The changes in fluorescence intensity at $465 \mathrm{~nm}$ were measured following the addition of cationic nanoparticles with an excitation wavelength of $405 \mathrm{~nm}$. 

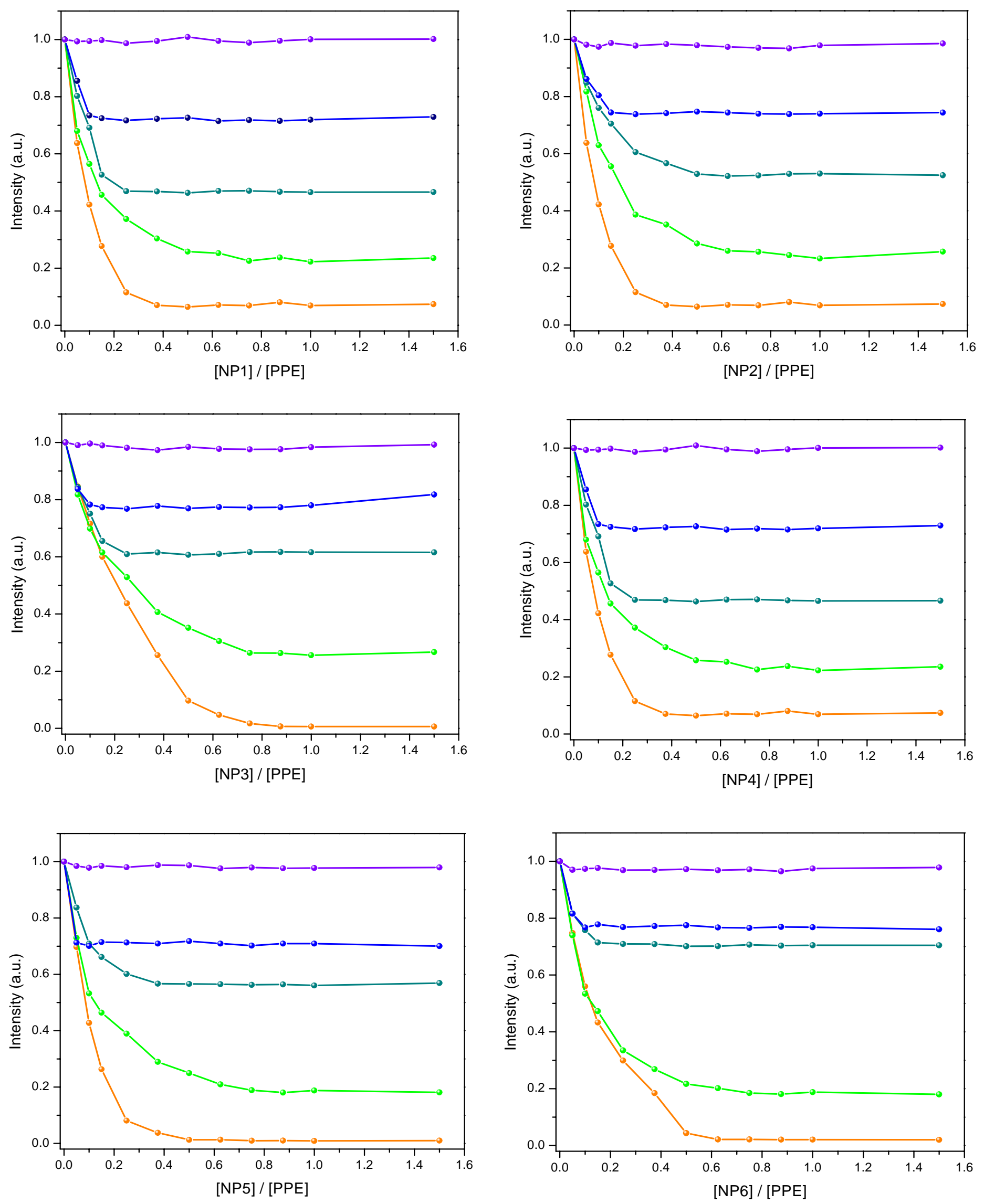

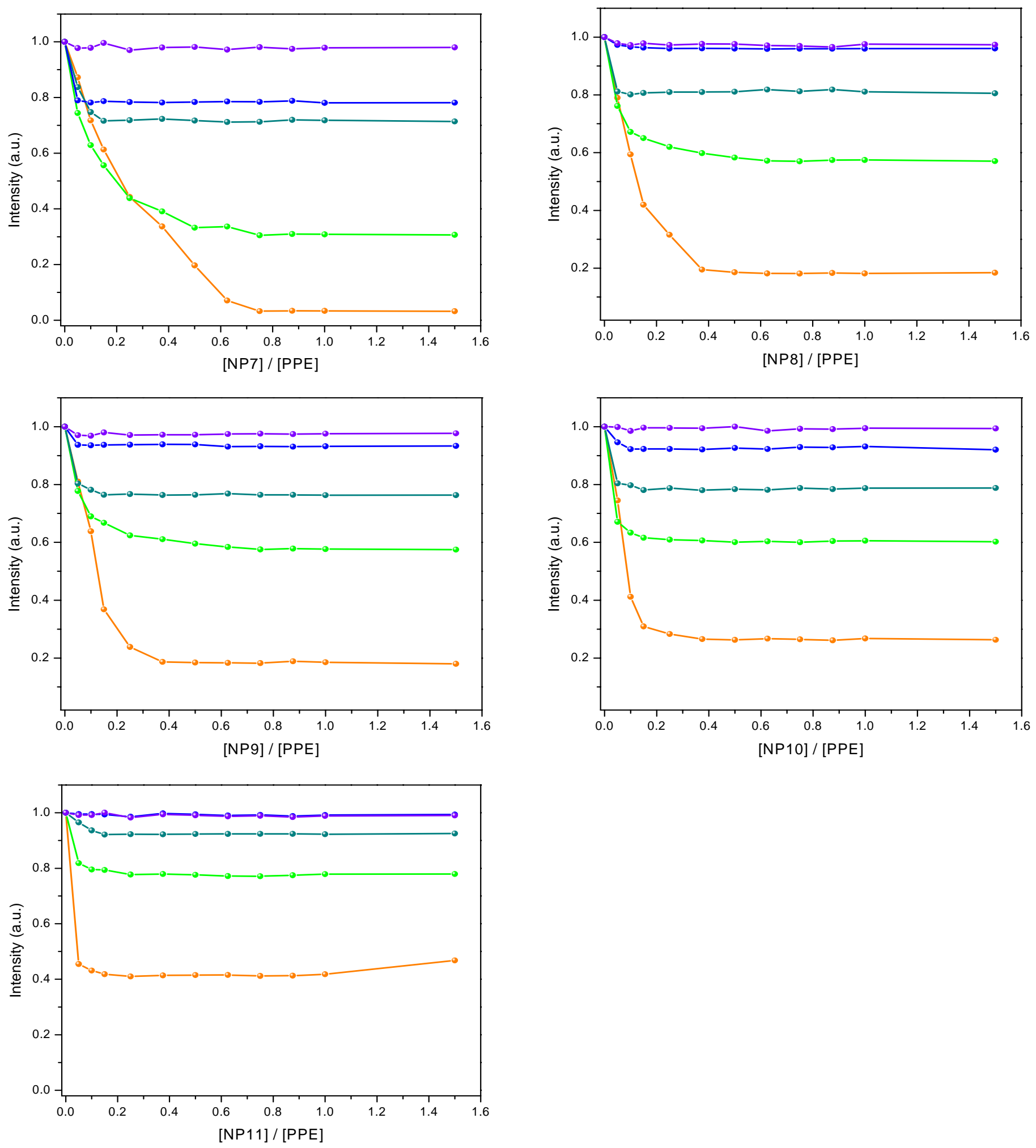

Figure S7. Overlap of fluorescence titration curves for the complexation of $\mathbf{N P}_{\mathbf{1}}-\mathbf{N P}_{\mathbf{1 1}}$ with Sw-CO $\mathbf{C O}_{2}$ in $\mathrm{PB}$ with $0,100,250,500$, and $1000 \mathrm{mM} \mathrm{NaCl}$. The changes in fluorescence intensity at $465 \mathrm{~nm}$ were measured following the addition of cationic nanoparticles with an excitation wavelength of $405 \mathrm{~nm}$. Legend: Orange $(0 \mathrm{mM} \mathrm{NaCl})$, green $(100 \mathrm{mM} \mathrm{NaCl})$, dark cyan $(250 \mathrm{mM} \mathrm{NaCl})$, blue $(500 \mathrm{mM} \mathrm{NaCl})$ and violet $(1000 \mathrm{mM} \mathrm{NaCl})$ 
Table S1. $\mathrm{K}_{\mathrm{a}}$ and $\Delta \mathrm{G}$ values for the complexation of $\mathbf{N P}_{\mathbf{1}}-\mathbf{N P}_{\mathbf{1 1}}$ with $\mathbf{S w}-\mathbf{C O}_{\mathbf{2}}$ in $\mathrm{PB}$ with 0 , $100,250,500$, and $1000 \mathrm{mM} \mathrm{NaCl}$. At higher $\mathrm{NaCl}$ concentrations, values could not be accurately determined for all nanoparticle-conjugated polymer constructs.

\begin{tabular}{|c|c|c|c|c|c|}
\hline Buffer & Nanoparticle & $\mathbf{n}$ & $\alpha$ & $\begin{array}{c}K_{a} \\
\left(M^{-1}\right) \\
\end{array}$ & $\begin{array}{c}\Delta \mathbf{G} \\
\left(\mathbf{k J} / \mathbf{K}^{*} \mathrm{~mol}\right) \\
\end{array}$ \\
\hline $\mathrm{PB}(\mathrm{OmM} \mathrm{NaCl})$ & 1 & 2.89 & $-1.07 \mathrm{E}+07$ & $7.46 \mathrm{E}+07$ & -44.6 \\
\hline $\mathrm{PB}(0 \mathrm{mM} \mathrm{NaCl})$ & 2 & 7.28 & $-1.02 \mathrm{E}+07$ & $7.58 \mathrm{E}+07$ & -44.6 \\
\hline $\mathrm{PB}(\mathrm{OmM} \mathrm{NaCl})$ & 3 & 4.61 & $-1.01 \mathrm{E}+07$ & 8.75E+07 & -45.0 \\
\hline $\mathrm{PB}(\mathrm{OmM} \mathrm{NaCl})$ & 4 & 3.65 & $-1.09 \mathrm{E}+07$ & $2.71 E+07$ & -42.1 \\
\hline $\mathrm{PB}(\mathrm{OmM} \mathrm{NaCl})$ & 5 & 5.71 & $-9.97 \mathrm{E}+06$ & $1.26 \mathrm{E}+08$ & -45.9 \\
\hline $\mathrm{PB}(0 \mathrm{mM} \mathrm{NaCl})$ & 6 & 10.4 & $9.93 \mathrm{E}+06$ & $1.85 \mathrm{E}+07$ & -41.1 \\
\hline $\mathrm{PB}(\mathrm{OmM} \mathrm{NaCl})$ & 7 & 12.9 & $-9.61 \mathrm{E}+06$ & $4.21 \mathrm{E}+07$ & -43.2 \\
\hline $\mathrm{PB}(0 \mathrm{mM} \mathrm{NaCl})$ & 8 & 5.96 & $9.43 \mathrm{E}+06$ & $1.09 \mathrm{E}+08$ & -45.5 \\
\hline $\mathrm{PB}(\mathrm{OmM} \mathrm{NaCl})$ & 9 & 6.14 & $-9.28 \mathrm{E}+06$ & $7.75 E+08$ & -50.4 \\
\hline $\mathrm{PB}(\mathrm{OmM} \mathrm{NaCl})$ & 10 & 5.98 & $-9.26 \mathrm{E}+06$ & $1.31 \mathrm{E}+09$ & -51.7 \\
\hline $\mathrm{PB}(\mathrm{OmM} \mathrm{NaCl})$ & 11 & 5.74 & $-9.24 \mathrm{E}+06$ & $1.04 \mathrm{E}+10$ & -56.8 \\
\hline PB (100mM NaCl) & 1 & 2.43 & $-9.11 E+06$ & $2.54 \mathrm{E}+06$ & -36.3 \\
\hline PB (100mM NaCl) & 2 & 6.84 & $-9.12 \mathrm{E}+06$ & $3.39 E+06$ & -37.0 \\
\hline PB (100mM NaCl) & 3 & 4.29 & $-9.11 E+06$ & $5.62 E+06$ & -38.2 \\
\hline PB (100mM NaCl) & 4 & 3.36 & $-9.08 \mathrm{E}+06$ & 8.37E+06 & -39.2 \\
\hline PB (100mM NaCl) & 5 & 5.49 & $-9.01 E+06$ & $6.24 \mathrm{E}+07$ & -44.2 \\
\hline PB (100mM NaCl) & 6 & 9.7 & $-8.98 \mathrm{E}+06$ & $7.91 \mathrm{E}+06$ & -39.1 \\
\hline PB (100mM NaCl) & 7 & 12.1 & $-8.73 E+06$ & 4.82E+06 & -37.9 \\
\hline PB (100mM NaCl) & 8 & 5.43 & $-8.61 \mathrm{E}+06$ & $9.70 \mathrm{E}+06$ & -39.6 \\
\hline PB (100mM NaCl) & 9 & 5.87 & $-8.49 \mathrm{E}+06$ & $1.60 \mathrm{E}+07$ & -40.8 \\
\hline PB (100mM NaCl) & 10 & 5.63 & $-8.47 \mathrm{E}+06$ & $3.09 E+08$ & -48.1 \\
\hline PB (100mM NaCl) & 11 & 5.48 & $-8.43 E+06$ & $8.57 \mathrm{E}+08$ & -50.6 \\
\hline $\mathrm{PB}(250 \mathrm{mM} \mathrm{NaCl})$ & 1 & 2.09 & $-7.97 \mathrm{E}+06$ & $2.47 E+05$ & -30.6 \\
\hline PB (250mM NaCl) & 2 & 6.37 & $-7.95 \mathrm{E}+06$ & $5.35 E+05$ & -32.5 \\
\hline PB (250mM NaCl) & 3 & 3.83 & $-7.99 \mathrm{E}+06$ & $7.21 \mathrm{E}+05$ & -33.2 \\
\hline $\mathrm{PB}(250 \mathrm{mM} \mathrm{NaCl})$ & 4 & 3.18 & $-7.92 \mathrm{E}+06$ & 4.97E+05 & -32.3 \\
\hline PB (250mM NaCl) & 5 & 4.98 & $-7.89 \mathrm{E}+06$ & $7.46 \mathrm{E}+06$ & -38.9 \\
\hline PB (250mM NaCl) & 6 & 9.25 & $-7.85 \mathrm{E}+06$ & $4.65 E+05$ & -32.1 \\
\hline PB (250mM NaCl) & 7 & 11.4 & $-7.69 \mathrm{E}+06$ & 1.38E+05 & -29.1 \\
\hline PB (250mM NaCl) & 8 & 5.09 & $-7.54 \mathrm{E}+06$ & $5.48 E+05$ & -32.5 \\
\hline PB (250mM NaCl) & 9 & 5.21 & $-7.47 \mathrm{E}+06$ & $6.13 \mathrm{E}+06$ & -38.4 \\
\hline $\mathrm{PB}(250 \mathrm{mM} \mathrm{NaCl})$ & 10 & 5.13 & $-7.42 \mathrm{E}+06$ & $1.39 \mathrm{E}+06$ & -34.8 \\
\hline $\mathrm{PB}(250 \mathrm{mM} \mathrm{NaCl})$ & 11 & 5.03 & --- & --- & --- \\
\hline
\end{tabular}




\begin{tabular}{|c|c|c|c|c|c|}
\hline Buffer & Nanoparticle & $\mathbf{n}$ & $\alpha$ & $\begin{array}{c}K_{a} \\
\left(M^{-1}\right)\end{array}$ & $\begin{array}{c}\Delta \mathbf{G} \\
\left(\mathrm{kJ} / \mathbf{K}^{*} \mathrm{~mol}\right)\end{array}$ \\
\hline $\mathrm{PB}(500 \mathrm{mM} \mathrm{NaCl})$ & 1 & 1.89 & $-7.29 \mathrm{E}+06$ & $2.21 \mathrm{E}+03$ & -19.0 \\
\hline $\mathrm{PB}(500 \mathrm{mM} \mathrm{NaCl})$ & 2 & 5.92 & $-7.28 \mathrm{E}+06$ & $3.27 E+03$ & -19.9 \\
\hline $\mathrm{PB}(500 \mathrm{mM} \mathrm{NaCl})$ & 3 & 3.51 & $-7.24 \mathrm{E}+06$ & 4.34E+03 & -20.6 \\
\hline $\mathrm{PB}(500 \mathrm{mM} \mathrm{NaCl})$ & 4 & 2.78 & $-7.22 \mathrm{E}+06$ & $3.09 E+03$ & -19.8 \\
\hline $\mathrm{PB}(500 \mathrm{mM} \mathrm{NaCl})$ & 5 & 4.46 & $-7.19 E+06$ & $7.29 E+03$ & -21.9 \\
\hline $\mathrm{PB}(500 \mathrm{mM} \mathrm{NaCl})$ & 6 & 8.84 & $-7.17 \mathrm{E}+06$ & $2.94 \mathrm{E}+03$ & -19.7 \\
\hline PB (500mM NaCl) & 7 & 10.3 & $-6.99 \mathrm{E}+06$ & $1.01 \mathrm{E}+03$ & -17.0 \\
\hline $\mathrm{PB}(500 \mathrm{mM} \mathrm{NaCl})$ & 8 & --- & --- & --- & --- \\
\hline $\mathrm{PB}(500 \mathrm{mM} \mathrm{NaCl})$ & 9 & --- & --- & --- & --- \\
\hline $\mathrm{PB}(500 \mathrm{mM} \mathrm{NaCl})$ & 10 & --- & --- & --- & --- \\
\hline $\mathrm{PB}(500 \mathrm{mM} \mathrm{NaCl})$ & 11 & --- & --- & --- & --- \\
\hline $\mathrm{PB}(1000 \mathrm{mM} \mathrm{NaCl})$ & 1 & --- & --- & --- & --- \\
\hline $\mathrm{PB}(1000 \mathrm{mM} \mathrm{NaCl})$ & 2 & --- & --- & --- & --- \\
\hline $\mathrm{PB}(1000 \mathrm{mM} \mathrm{NaCl})$ & 3 & --- & --- & --- & --- \\
\hline $\mathrm{PB}(1000 \mathrm{mM} \mathrm{NaCl})$ & 4 & --- & --- & --- & --- \\
\hline PB (1000mM NaCl) & 5 & --- & --- & --- & --- \\
\hline PB (1000mM NaCl) & 6 & --- & --- & --- & --- \\
\hline PB (1000mM NaCl) & 7 & --- & --- & --- & --- \\
\hline PB (1000mM NaCl) & 8 & --- & --- & --- & --- \\
\hline PB (1000mM NaCl) & 9 & --- & --- & --- & --- \\
\hline PB (1000mM NaCl) & 10 & --- & --- & --- & --- \\
\hline PB (1000mM NaCl) & 11 & --- & --- & --- & --- \\
\hline
\end{tabular}



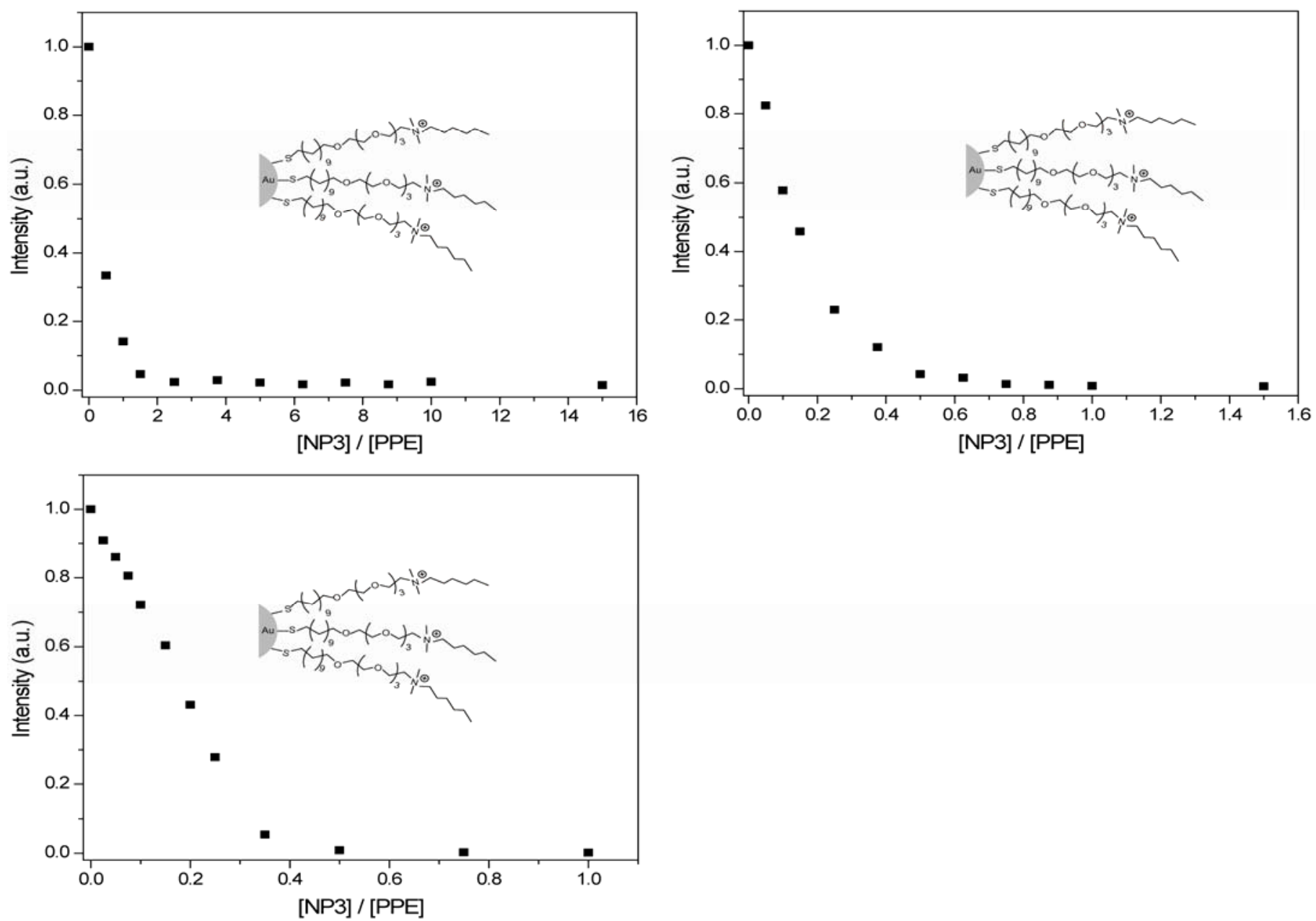

Figure S8. Fluorescence titration curves for the complexation of $\mathbf{N P}_{\mathbf{3}}$ with $10 \mathrm{nM}$ (top left), $100 \mathrm{nM}$ (top right), and $1000 \mathrm{nM}$ (bottom left) Sw-CO $\mathbf{O}_{2}$. The changes in fluorescence intensity at $465 \mathrm{~nm}$ were measured following the addition of cationic nanoparticles with an excitation wavelength of $405 \mathrm{~nm}$. 
Table S2. $\mathrm{K}_{\mathrm{a}}$ and $\Delta \mathrm{G}$ values for the complexation of $\mathbf{N P}_{3}$ with 10,100 , and $1000 \mathrm{nM} \mathbf{S w}-$ $\mathrm{CO}_{2}$ in $\mathrm{PB}$.

\begin{tabular}{|c|c|c|c|}
\hline $\begin{array}{c}\text { [PPE] } \\
(\mathbf{n M})\end{array}$ & $\mathbf{N P}$ & $\begin{array}{c}\mathbf{K}_{\mathbf{a}} \\
\left(\mathbf{M}^{-1}\right)\end{array}$ & $\begin{array}{c}\text { Delta G } \\
\left(\mathbf{k J} / \mathbf{K}^{*} \mathbf{m o l}\right)\end{array}$ \\
\hline 10 & 3 & $8.63 \mathrm{E}+07$ & -45.0 \\
\hline 100 & 3 & $8.75 \mathrm{E}+07$ & -45.0 \\
\hline 1000 & 3 & $8.51 \mathrm{E}+07$ & -44.9 \\
\hline
\end{tabular}

\section{References:}

1. Kim, I.B.; Phillips, R.L.; Bunz, U.H.F. Macromolecules 2007, 40, 5290-5293.

2. You, C.-C.; Miranda, O.R.; Ghosh, P.S.; Kim, I.B.; Erdogan, B.; Krovi, S.A.; Bunz, U.H.F.; Rotello, V.M. Nat. Nanotechnol. 2007, 2, 318-323.

3. Brust, M.; Walker, M.; Bethell, D.; Schiffrin, D.J.; Whyman, R. J. Chem. Soc., Chem. Commun. 1994, 801-802.

4. Hostetler, M.J.; Templeton, A.C.; Murray, R.W. Langmuir 1999, 15, 3782-3789. 